\title{
Procesos de cobranza y morosidad en una empresa de servicios de marketing
}

Collection and delinquency processes in a marketing services company

Processos de cobrança e inadimplência em uma empresa de serviços de marketing

\author{
Nieves del Pilar Pizzán-Tomanguillo \\ nievesdelpilarpizzan1993@gmail.com \\ CNL ASESORES - Perú \\ ORCID: https://orcid.org/0000-0001-9933-8738
}

Erica Enid García Delgado ericaenid_garcía@hotmail.com

Universidad César Vallejo - Perú

ORCID: https://orcid.org/0000-0003-3044-0886

Carlos Daniel Rosales Bardalez

bardalez.daniel@gmail.com

Universidad Peruana Unión - Perú

ORCID: https://orcid.org/0000-0002-2311-1577

\author{
Sandra Lucero Pizzán-Tomanguillo \\ 1u19930823@gmail.com \\ CNL ASESORES - Perú \\ ORCID: https://orcid.org/0000-0001-5124-9154
}

\begin{abstract}
RESUMEN
El presente estudio tuvo como objetivo general determinar la incidencia de los procesos de cobranzas en la morosidad de la empresa Digital San Martín S.A.C. de Tarapoto. El estudio ha presentado un diseño no experimental con corte transversal, nivel explicativo y para el análisis de datos se empleó el método hipotético-deductivo. En la evaluación de los procesos de cobranza desarrollados durante el 2020, se utilizaron como instrumentos la guía de entrevista y guía de análisis documental. Resultados: El 100\% de acciones de los procesos de cobranza no son efectuadas eficiente ni oportunamente. Todo ello permitió evidenciar un $84 \%$ de morosidad y 39\% de cobertura, ambos índices suscitados por el aumento del importe de las cuentas pendientes de pago representado por S/135006. Así también, la empresa cuenta con registros e información documentaria no actualizada; no muestra estrategias de cobro acorde con su realidad; carece de un monitoreo organizado y sistemático del importe total de la deuda pendiente de pago; presenta un deficiente desarrollo de las actividades de control de deudas, entre otros. Conclusión: los procesos de cobranza inciden negativamente en la morosidad de la empresa.
\end{abstract}

Palabras clave: procesos, estrategias, cobranza, morosidad, servicios

\begin{abstract}
The general objective of this study was to determine the incidence of collection processes in the delinquency of the company Digital San Martín S.A.C. from Tarapoto. The study has presented a non-experimental cross-sectional design, explanatory level, and the hypothetical-deductive method was used for data analysis. In the evaluation of the collection processes developed during 2020, the interview guide and the documentary analysis guide were used as instruments. Results: $100 \%$ of the actions of the collection processes are not carried out efficiently or in a timely manner. All this made it possible to show $84 \%$ delinquency and $39 \%$ coverage, both indexes caused by the increase in the amount of accounts pending payment represented by S/135006. Also, the company has non-updated records and documentary information; does not show collection strategies in accordance with their reality; lacks organized and systematic monitoring of the total amount of outstanding debt; it presents a poor development of debt control activities, among others. Conclusion: the collection processes negatively affect the delinquency of the company.
\end{abstract}

Keywords: Processes, strategies, collection, delinquency, services

RESUMO 
O objetivo geral deste estudo foi determinar a incidência de processos de cobrança na inadimplência da empresa Digital San Martín S.A.C. de Tarapoto. O estudo apresentou delineamento transversal não experimental, nível explicativo, e utilizou-se o método hipotético-dedutivo para análise dos dados. $\mathrm{Na}$ avaliação dos processos de coleta desenvolvidos ao longo de 2020, foram utilizados como instrumentos o roteiro de entrevista e o roteiro de análise documental. Resultados: $100 \%$ das ações dos processos de cobrança não são realizadas de forma eficiente ou tempestiva. Tudo isso possibilitou evidenciar $84 \%$ de inadimplência e $39 \%$ de cobertura, ambos índices decorrentes do aumento da quantidade de contas pendentes representadas pelo S/135006. Além disso, a empresa possui registros e informações documentais não atualizados; não apresenta estratégias de cobrança de acordo com sua realidade; carece de acompanhamento organizado e sistemático do montante total da dívida pendente; apresenta um fraco desenvolvimento das atividades de controle da dívida, entre outras. Conclusão: os processos de cobrança afetam negativamente a inadimplência da empresa.

Palavras-chave: processos, estratégias, cobrança, inadimplência, serviços

\section{INTRODUCCIÓN}

Actualmente, las organizaciones que se dedican a prestar servicios relacionados con la publicidad y marketing buscan diseñar y aplicar nuevas estrategias de promoción, pasando por alto los procedimientos apropiados respecto a la cobranza, lo cual conlleva a que se presente un incremento considerable de la cartera de clientes morosos. Por esta razón, se considera que los procedimientos orientados a efectuar la cobranza tienen que estructurarse en función al contexto real y a las operaciones que realiza la empresa, siendo indispensable, además, que estos procedimientos estén en constante monitoreo y evaluación permanente para certificar la obtención de buenos resultados.

De esta manera, Guillén y Peñafiel (2018) explican la importancia que implica que el personal que participa en cada uno de estos procedimientos de cobranzas cuente con la capacitación y conocimiento suficiente de modo que se pueda detener de forma oportuna el incremento del reporte de cuentas pendientes de cobro, así también, el compromiso para cumplir con cada uno de los lineamientos y pautas estipuladas, por cuanto su inobservancia representaría un riesgo significativo para el desempeño económico de la organización. De igual manera, Landeta e Ynzunza (2017) sostienen que las organizaciones que prestan servicios de marketing buscan continuamente aumentar su cartera de clientes y ello ha conllevado a que el importe de sus cuentas por cobrar crezca de forma acelerada, además, el 63\% de estas organizaciones utilizan estrategias flexibles de cobro dadas a través de llamadas o mensajes, que suelen no incidir positivamente en la recuperación del efectivo.

En Perú, considerando la información dada por la Cámara de Comercio, se registró un incremento considerable de la morosidad en las organizaciones prestadoras de servicio, alcanzando un $5,1 \%$ en los seis primeros meses del 2020 , lo cual representó una tasa de incumplimiento más elevada en el transcurso del último lustro (Rodrigues y Florencio, 2020). De esta forma, el estudio efectuado por Magallanes (2019), en una de las empresas de marketing más prestigiosas del país (MK agencias) refleja diversas irregularidades que facilitó el incrementó de la morosidad como la falta de aplicación de herramientas y técnicas eficientes para monitorear continuamente los pagos e impedir que el importe de cuentas incobrables aumente.

En tanto, adentrando a una realidad de la empresa Digital San Martín S.A.C., después de una evaluación detallada de los registros mensuales de las cuentas por cobrar y ventas realizadas durante el periodo 2020, se ha percibido que los colaboradores no disponen de las herramientas y acciones estratégicas que limiten el aumento continuo de la cartera de cuentas pendientes de cobro; así también, más del 50\% de los mismos, no tienen un conocimiento claro de las políticas y procedimiento en el otorgamiento del servicio bajo la modalidad de crédito. Así también, la entidad dispone de un software mediante el cual se identifiquen las cuentas con fecha de vencimiento próximo para que los colaboradores actúen de forma proactiva y procedan con el cobro respectivo; igualmente, no se usa un sistema de cobro para la notificación y castigo de los clientes morosos. Así 
también, García \& Pizzan (2020) señalan que la empresa no dispone con recursos humano que oriente de manera detallada y claro la aplicación de las estrategias, además, la falta de revisión y seguimiento de las deudas que están por vencer ha conllevado a maximizar la cartera de clientes morosos.

En tal sentido, se formuló como problema general la siguiente pregunta: ¿Cómo inciden los procesos de cobranza en la morosidad de la empresa Digital San Martín S.A.C.?, como problemas específicos los siguientes: ¿Cuáles son los procesos de cobranza en la empresa Digital San Martin S.A.C. de Tarapoto?, ¿Cuáles son las deficiencias en los procesos de cobranza en la empresa Digital San Martin S.A.C. de Tarapoto?, y ¿Cómo es la morosidad en la empresa Digital San Martin S.A.C. de Tarapoto? Siendo así, resultó como objetivo general determinar la incidencia de los procesos de cobranza en la morosidad de la empresa Digital San Martín S.A.C. de Tarapoto. Y los específicos son: Describir los procesos de cobranza en la empresa Digital San Martin S.A.C. de Tarapoto; Identificar las deficiencias en los procesos de cobranza en la empresa Digital San Martin S.A.C. de Tarapoto, y Evaluar la morosidad en la empresa Digital San Martin S.A.C. de Tarapoto.

Al mismo tiempo se presentan las justificaciones que comprenden las razones y motivos de la realización del estudio, desde un concepto de relevancia social, con los resultados obtenidos en la investigación, será de beneficio a las empresas y personas interesadas de conocer la importancia de contar con eficientes recursos y procesos de cobranzas, y como estas actividades se relacionan con el incremento de los índices de morosidad. Por lo que refiere al valor teórico, en la determinación del estudio fue importante la utilización de información relevante y veraz en el desarrollo de los objetivos planteados en la investigación, la misma que permitirá el acrecentamiento de conocimiento en cuanto a las variables en estudio, para ello se ha considerado autores que definan características, cualidades y el proceder de estas. En cuanto, a la implicancia práctica, con la obtención de los resultados, se ha informado a los responsables de la empresa en evaluación de los hechos y sucesos que viene originando la inadecuada administración de las cuentas por cobrar y el incremento de la morosidad, esto con la finalidad de establecer estrategias, técnicas y métodos que coadyuvan al crecimiento económico de la empresa.

Para el estudio se formuló como hipótesis general Hi: Los procesos de cobranza inciden de manera negativa en la morosidad de la empresa Digital San Martín S.A.C. de Tarapoto. Las hipótesis especificas son: Hi1: Los procesos de cobranza en la empresa Digital San Martin S.A.C. de Tarapoto son el conocimiento del cliente, identificación de la capacidad de pago, seguimiento de pagos y aplicación de las estrategias de cobranza, Hi2: Las deficiencias en los procesos de cobranza en la empresa Digital San Martin S.A.C. de Tarapoto son la falta de aplicación de la identificación del riesgo y estrategias de cobranzas; Hi3: La morosidad en la empresa Digital San Martin S.A.C. de Tarapoto no es óptima.

En la investigación se desarrolla en el apartado dos el fundamento teórico, en la que se muestra las investigaciones relacionadas a las variables en estudio, como también la base teórica de las variables y dimensiones, por otro lado en el apartado tres, se desarrolla los procedimientos metodológicos, donde se describe la metodología utilizada, las técnicas e instrumentos aplicados en la investigación, en el apartado cuatro, se desarrollan los resultados y discusiones, se presenta los resultados en forma narrativa y grafica de acuerdo a los objetivos planteados, se realiza un análisis crítico sobre las mismas, para posteriormente comparar los mismos con otros estudios similares, sigue las conclusiones, se presenta en forma resumida la respuesta al objetivo del estudio, como se legró a lo que se llegó, también mostrar las limitaciones y sugerir futuras investigaciones, por último se muestra las referencias utilizadas en el desarrollo del artículo.

\section{FUNDAMENTO TEÓRICO}

\subsection{Antecedentes}


Para el abordaje del estudio fue necesario analizar antecedentes como el de García, Galarza y Grijalva (2019), quienes concluyeron que un 33,3\% de las operaciones de cobranzas no están siendo realizadas eficientemente, visto que los colaboradores efectúan sus labores empíricamente y el $83 \%$ del personal no aplican pautas ni lineamientos de cobro o crédito, ocasionando que las cuentas no se recuperen dentro de los plazos de tiempo previstos de acuerdo con lo estipulado en el acuerdo por la contratación del servicio.

Miranda (2018), en su trabajo de investigación llego a concluir, que las estrategias de cobranza no se encuentran diseñadas de acuerdo con la realidad de la empresa, puesto que existe ciertos vacíos que dan lugar al incumplimiento de pagos, por ende, esta es de 56\% deficiente. En cuanto a la morosidad presenta un nivel medio en un $49 \%$, las mismas que vienen afectando de manera significativa el cumplimiento de las obligaciones con los colaboradores y trabajadores de la empresa, ya que se observa continuas quejas por incumplimiento de pagos, y es importante mencionar que la empresa aún no ha diseñado y mejorado las estrategias de cobranzas. Por lo tanto, existe relación entre la estrategia de cobranza y la morosidad por cuanto la estadística correlacional presenta un valor de (0.696), el cual indica una correlación positiva media, asimismo el valor de la significancia bilateral fue de (0.001), por lo tanto, se acepta la hipótesis alterna de la investigación (p.10).

Seguidamente, Castillo (2018) en su llego a concluir los proveedores recurrentes son aquellos que reciben mayores cantidades de productos a créditos, los mismo que al proporcionar los productos, se cobran de ello, esta variable, ha sido evaluada regular en un $74 \%$, Por lo que refiere al nivel de morosidad esta presenta un nivel medio en un 58\%, por cuanto se evidencia el incumplimiento de pagos por parte de los clientes. Finalmente, se evidencia que existe relación entre la gestión de cobranzas y la morosidad, por cuanto los resultados estadísticos Chi refleja un valor (0.721), con una significancia bilateral de (0.001), el cual indica una correlación positiva considerable entre las variables en estudio, por ente se acepta la hipótesis alterna de la investigación

Por lo siguiente, Guevara y Upiachihua (2018) en su trabajo de investigación titulado “Gestión cobranza y morosidad de la empresa Televisión SAC. Tarapoto, año 2017”. Llegaron a concluir que tras las evaluaciones en cuanto a los procesos de cobranza se conoció que estos son inadecuados en un $67.37 \%$, Al tratar de la variable morosidad, esta presenta un $37.5 \%$ en índices, la misma que es considerada como un alto y significativo indicador. Por lo que se puede converger que la gestión de cobranzas incide de manera negativa en la morosidad.

Ramos (2020) en su investigación sobre la morosidad y sus causas concluyo que el $46 \%$ de las entidades financieras cuentan con un control inapropiado, esto ha permitido que el riesgo crediticio incremente de manera trascendental, las misma que ha puesto en riesgo a la entidad. Por otro lado, es importante mencionar que el procedimiento de otorgamiento de crédito no es eficiente opinaron el $13 \%$, puesto que no existe una previa evaluación del usuario, en el que se solicite los documentos necesarios para acreditar la capacidad de pago, asimismo algunos de los requisitos solicitados al cliente, no se aplican, por la afinidad que presenta con el analista de crédito.

Por su parte, Royo (2013) determinó que las organizaciones situadas en Alcalá (España) adoptan medidas de cobranza rígida, en aquellos casos en los que las cuentas no puedan recuperarse fácilmente; presentándose en tales casos un índice equivalente a 0,85 de morosidad, siendo necesario que se realicen ventas de la deuda a los agentes de cobro de mayor tamaño.

Finalmente, Campodónico (2019) señalo que las actividades efectuadas en las cobranzas son aquellas que involucran el análisis de la cartera de clientes, la evaluación contundente de las estrategias de cobranza y el seguimiento de pago, los mismos que son desarrollados de manera deficiente de 63\%. Los índices rentables y de liquidez son inferiores a los obtenidos a los años anteriores. Puesto que la liquidez obtenida en la empresa es de S/ 152 196.03. cuyo ratio obtenido es de 1.22, por ello se acepta que ambas variables se relación de manera negativa, puesto que su dependencia influyó de manera negativa entre ambas. 


\subsection{Procesos de cobranza}

Desde un enfoque teórico, Lionel (2018) y Bengolea y Pérez (2016) refieren que los procesos de cobranza son operaciones que tienen como fin captar una deuda cuya fecha de vencimiento ya caducó o está próxima a caducar, ejecutadas ordenadamente con el fin de asegurar el recaudo oportuno y efectivo de la cuenta incobrable. De igual manera, López (2019) señala que son acciones enfocadas a recuperar una deuda, sin necesidad de afectar la estabilidad del cliente, para ello es importante ser minucioso en la ejecución de acciones radicales que conlleven a una limitación al momento de cancelar las deudas. Adicionalmente, Mauad (2017) sostiene que dichos procesos abarcan actividades que buscan reactivar comercialmente a la organización y los clientes con la finalidad de mantener la cancelación de sus deudas en las fechas que correspondan.

En efecto, la gestión de cobro tiene que estar constituida por medidas estratégicas idóneas que coadyuven con la optimización del desempeño económico de la organización y reducción del nivel de mora. Cabe resaltar que estas operaciones van a contribuir a que las cuentas vencidas se conviertan en un activo líquido, mejorando el flujo de caja y el funcionamiento de la organización (Gonzáles, Leal, Martínez y Morales, 2019; Guizar, González y Ezzahra, 2020).

Por su parte, Do Prado, Melo, Carvalho y Ribeiro (2019) fundamentan que el proceso de cobranza busca cumplir una serie de propósitos específicos, como asegurar que el cliente cancele en las fechas previstas el importe de la obligación contraída sin necesidad de que ello afecte el vínculo que existe entre ambas partes (organización y cliente) y analizar el grado de flexibilidad, en caso hubiese, del vínculo de naturaleza comercial-económica de la organización.

Para estimar estos procesos de cobranzas, fue necesario la aplicación de la teoría de Lionel (2018), quien menciona que son actividades destinadas a la recuperación de las deudas, para ello es importante ejecutar la identificación del riesgo, en este proceso se pretende conocer el comportamiento del cliente, asimismo prevenir actitudes que manifiestan anticipadamente la incobrabilidad del pago. Es por ello, por lo que se ejecuta una serie de acciones que permitirán lograr la identificación del riesgo, como se muestra a continuación: el conocimiento del cliente, la identificación de la capacidad de pago, y el seguimiento de pagos.

Como segundo proceso es la aplicación de las estrategias de cobranzas, Lionel (2018), alude que cuando se ha verificado y constatado el incumplimiento de las cuotas pactadas con el cliente, se procede a ejecutar acciones de cobranzas, las mismas que garantizas la cobrabilidad de las cuotas vencidas, que a continuación se detalla: mensajes, llamadas, notificaciones y sistemas de cobranza.

\subsection{Morosidad}

Referente a la morosidad, esta es la ausencia de cumplimiento del pago de una deuda u obligación celebrada anteriormente (Ferro, 2014), por lo cual, se ha generado un interés adicional en base a una tasa aplicable por la cantidad de días que han transcurrido después de haberse vencido tal obligación. Frente a este hecho, las organizaciones creen conveniente proceder con una acción legal para recuperar la deuda (Gonzalo, 2012); sin embargo, resulta necesario precisar también que la morosidad podría representar un gasto adicional para una organización en la medida en la que limite su desarrollo, desde una perspectiva económica (Romero, 2017; López, 2006), por lo que no resulta una medida totalmente oportuna para la empresa.

Tal como lo señala, Ferro (2020), la morosidad es evaluada a través elementos de clasificación y ratios que permiten conocer el índice de morosidad. En cuanto a la clasificación según los días de morosidad, existen cinco (5) clasificaciones entre ellas tenemos: Normal: Son aquellos clientes que efectúan sus pagos de manera puntual o presentan retrasos de ocho (8) días calendarios., seguidamente tenemos los potenciales, son aquellos clientes que demuestran retrasos 
en el pago de sus deudas de entre 9 a 30 días., por consiguiente los deficientes, son aquellos que presentan retrasos entre 31 a 60 días calendarios, así también, tenemos a los clientes con clasificación dudoso, quienes presentan retrasos en el pago de entre 61 a 120 días calendarios, y por ultimo tenemos a los clientes que se clasifican como pérdida, son aquellos clientes que presentan retrasos en el pago de más de 120 días calendarios, siendo estos considerados como pérdida. Así también se empleará indicadores de morosidad, que se señalan a continuación: Índice de morosidad: el presente detalla la cantidad de las cuentas por cobrar vencidas, deudas mayores a 30 días, relacionadas al total de los servicios otorgados, expresado en soles, la misma que se emplea como formula la siguiente: Cuentas por cobrar vencidas / Total deuda por cobrar., También tenemos el índice de cobertura, la misma que evalúa las cuentas estimadas como gastos con respecto al total del servicio expresado en soles (Ferro, 2020), Cuya fórmula es la siguiente: Estimación de cobranzas dudosa / Total deuda por cobrar.

\section{PROCEDIMIENTOS METODOLOGICOS}

\subsection{Tipo y diseño}

El estudio ha presentado un tipo de investigación aplicada de enfoque cualitativa. Basándose en que se busca profundizar e incrementar conocimientos acerca de la realidad, así también, se pretende describir, comprender y explicar mejor los fenómenos o acontecimientos (Arbaiza, 2019).

\subsection{Población y muestra}

Los elementos de la población fueron los procesos de cobranza efectuados a lo largo del periodo 2020. En los criterios de inclusión se consideraron aquellos procesos dirigidos a los clientes que se encuentran calificados como "potencial", "deficiente", "dudoso" y "pérdida", acorde con los informes del mismo año. En los criterios de exclusión, se consideraron aquellos procesos dirigidos a los clientes que se encuentran calificados como "normal", es decir, quienes tienen un plazo de retraso entre dos y nueve días.

La muestra estuvo representada por los procesos de cobranza efectuados en el periodo 2020, y en ese sentido el muestreo aplicado fue intencional por conveniencia, debido a que el investigador escogió los elementos muestrales en base a sus criterios individuales para facilitar la consecución de los objetivos planteados.

El tipo de muestreo que se empleó fue el no probabilístico por conveniencia, ya que se trabajó con los individuos que aceptaron formar parte del estudio, lo que quiere decir que se les solicitó a los sujetos que participen voluntariamente, estando la decisión de formar parte de la muestra en la persona invitada, siempre y cuando haya cumplido con los criterios de inclusión.

\subsection{Técnica e instrumento}

En primera instancia, la técnica empleada fue la observación, entrevista y análisis documental; acorde con ello se tuvo que utilizar como instrumentos una lista de cotejo cuya escala de medición fue nominal ( $\mathrm{Si}$ y No), compuesta por 11 enunciados, tiene como finalidad identificar las deficiencias de los procesos de cobranzas, la misma que fue llenada por los investigadores para captar sucesos significativos donde se evidenciaron las irregularidades como parte del proceso de cobranza.

De igual manera, se utilizó una guía de entrevista constituida por quince (15) interrogantes formuladas con el objeto de conocer detalladamente todas las acciones ejecutadas dentro del marco del proceso de cobranza; la persona encargada de responder cada una de estas fue el jefe del área de cobranzas, por cuanto este cuenta con las aptitudes e información necesaria requerida para el recojo de los datos cualitativos. 
También, se utilizó una guía de análisis documental, con una escala razón (\%), la cual permitió recolectar la cantidad de clientes que se encuentran en la condición de morosos y el índice de morosidad alcanzado.

\subsection{Procedimiento}

De la recopilación de datos a través de los instrumentos, se procesaron mediante hojas excel, asimismo se obtuvo tablas para la representación gráfica, el cual nos permitió el desarrollo de la discusión de nuestro estudio, y luego las conclusiones.

Para el análisis de las variables se empleó a nivel descriptivo. García, A. (2018), este método consiste esencialmente en el estudio de las distribuciones de las variables, permite captar un perfil de los elementos o muestra en cuanto a su caracterización y cualidades.

En la investigación se aplicaron principios de beneficencia, justicia, autonomía, por cuanto se respetó la libre participación de las personas involucradas en la ejecución de la investigación, por tal motivo, estos fueron previamente informados sobre el proceso que se realizó con la finalidad de que brinden su consentimiento.

\section{RESULTADOS Y DISCUSIÓN}

\subsection{Procesos de cobranza en la empresa Digital San Martín S.A.C. de Tarapoto}

Al aplicar una guía de entrevista al gerente, se conocieron las acciones desarrolladas como parte de los procesos de cobranza, tal como se describe a continuación.

\section{Paso 1. Conocimiento del cliente}

El gerente tiene un diálogo con el cliente para adquirir información sobre la actividad que lleva a cabo o el rubro de su negocio, esto con el fin de direccionar las acciones estratégicas de marketing a aplicar; también se determina el paquete del servicio, el costo y medio de desembolso. Cabe mencionar que la organización cuenta con un brochure para que el cliente se ajuste en función a sus medios económicos, no obstante, usualmente los clientes eligen los paquetes más completos cuyo costo es superior.

\section{Proceso 2. Identificación de la capacidad de pago}

Seguidamente, el responsable determina el cronograma de pago previo consenso con los clientes y, dado que la cartera de clientes se constituye por personas cercanas y recomendadas, no se averigua su capacidad crediticia, y se procede a detallar las condiciones y responsabilidades de las partes en el contrato debidamente firmado.

\section{Paso 3. Seguimiento de pagos}

En este apartado, el personal responsable procede a efectuar la cobranza y revisar el estado de las cuentas empresariales para constatar el abono correspondiente; no obstante, al no evidenciar el depósito, se aplica la estrategia de cobranza y se suspende la prestación del servicio hasta que realice el pago correspondiente.

\section{Paso 4. Aplicación de las estrategias de cobranza}

La estrategia aplicada en un primer momento es el envío de mensajes. Para aquellos clientes categorizados como "normales" acorde con el tiempo de retraso que varía entre 8 días calendario o 
se encuentran registrados con la denominación de clientes puntuales, se remite un mensaje de forma personalizada; para aquellos categorizados bajo la denominación de "potenciales", cuyo tiempo de retraso varía entre 9 a 30 días calendario, se efectúa una llamada o se remite una carta personalizada señalando el importe de la deuda e interés. Para aquellos clientes categorizados como "deficientes, dudosos, pérdida", cuyo tiempo de retraso varía entre 31 a 120 días calendario, se emite una carta personalizada o se notifica señalando el importe de la deuda e interés. Además, se localiza el sistema de cobranza y se adquiere un paquete de castigo de 11 dólares estadounidenses para registrar al cliente, procediendo a provisionar como cuentas incobrables.

$\mathrm{Al}$ aplicar la lista de cotejo, se llegó a evidenciar que el 100\% de las acciones o procesos se llevan a cabo de manera inoportuna ni eficiente, a raíz de la falta de normativas y lineamientos que orienten las acciones que deben ejecutarse para realizar el cobro correspondiente de las deudas pendientes a los clientes morosos. Además, se reconoce que, como parte del proceso de cobro, se aplicaron ciertas acciones estratégicas de cobranza entre las cuales destacan los mensajes, llamadas, cartas personalizadas, correos, etc.; no obstante, tales estrategias no se realizaron oportunamente, trayendo como consecuencia retrasos en la recepción de los pagos.

Cabe mencionar que, García et al. (2019) concuerdan con los resultados del estudio, pues también concluyen que se genera un incremento de las cuentas incobrables debido a que un 33,3\% de las operaciones no se ajustan al plan de trabajo de la entidad, así también, el gerente no desempeña sus labores eficientemente, ya que el personal cumple con sus labores asignadas de modo empírico; mientras que el $83 \%$ no aplican los lineamientos asociados al crédito y cobranza. Asimismo, la documentación y reportes de los cobros realizados no se elaboran y actualizan continuamente porque el 56\% asegura que el plazo para su presentación es muy breve. Bajo este contexto, se reconoce la relevancia en la aptitud y capacidad del personal responsable del cobro de las cuentas dentro de una organización; es decir, debe poseer conocimiento sobre las medidas estratégicas para desarrollar los procesos apropiadamente, disponiendo también los medios esenciales que faciliten la recuperación de las cuentas pendientes de cobro.

\subsection{Deficiencias en los procesos de cobranzas en la empresa Digital San Martín S.A.C.}

Para identificar las deficiencias, fue necesario determinar si se cumplían las actividades de los procesos de cobranza, previa aplicación de la lista de cotejo (Ver Tabla 1).

Tabla 1

Cumplimiento de las actividades de procesos de cobranza

\begin{tabular}{|c|c|c|c|}
\hline \multirow{2}{*}{ Dimensiones } & \multirow{2}{*}{ Indicadores } & \multicolumn{2}{|c|}{$\begin{array}{l}\text { Cumplimiento de } \\
\text { actividades }\end{array}$} \\
\hline & & Sí & No \\
\hline \multirow{3}{*}{$\begin{array}{l}\text { Identificación del } \\
\text { riesgo }\end{array}$} & Conocimiento del cliente & 3 & $27 \%$ \\
\hline & Identificación de la capacidad de pago & 2 & $18 \%$ \\
\hline & Seguimiento del pago & 1 & $9 \%$ \\
\hline \multirow{5}{*}{$\begin{array}{l}\text { Aplicación de las } \\
\text { estrategias }\end{array}$} & Mensaje & 2 & $18 \%$ \\
\hline & Llamada & 1 & $9 \%$ \\
\hline & Notificación & 1 & $9 \%$ \\
\hline & Sistema de cobranza & 1 & $9 \%$ \\
\hline & Total & 11 & $100 \%$ \\
\hline
\end{tabular}

Nota: Elaboración propia con base en datos de la empresa Digital San Martín S.A.C. de Tarapoto

Todas las actividades presentadas no fueron desarrolladas eficientemente, es por ello que referente al conocimiento del cliente, existe un incumplimiento de las actividades en un $27 \%$ debido a que el personal competente en ningún momento o circunstancia solicita un reporte para analizar la realidad del cliente, desde un punto de vista económico, a fin de prevenir un posible riesgo significativo. Del mismo modo, sobre la Identificación de la capacidad de pago, un $18 \%$ de las 
actividades tampoco se cumple y solo se remiten mensajes. Esto conlleva a que las actividades de pago y modalidades de cobranza no estén siendo cumplidas en un $9 \%$.

Todo ello permite deducir que no hay una buena administración y organización de las atribuciones por parte de la alta dirección de la organización, lo cual conlleva a que se ponga en manifiesto la escasez de aptitudes sobre la eficacia de los procesos a seguir para reducir un aumento de las cuentas incobrables y contribuir con la obtención de fondos necesarios para la prestación de servicios de forma pertinente y buen rendimiento. En ese sentido, Miranda (2018) coincide con los resultados del estudio, pues deduce que las herramientas empleadas para el cobro de las deudas pendientes no fueron diseñadas considerando el contexto en el que se encuentra la organización, dado que se presenta un alto nivel de incumplimiento del pago en las fechas acordadas, llegando a ser deficientes en hasta un $56 \%$.

Tabla 2

Incremento de las cuentas por cobrar e incobrables

\begin{tabular}{lcc}
\hline Trimestre & Cuentas por cobrar 2020 & Cuentas incobrables \\
\hline Primer trimestre & $\mathrm{S} / 28521$ & $\mathrm{~S} / 4210$ \\
Segundo trimestre & $\mathrm{S} / 34517$ & $\mathrm{~S} / 8414$ \\
Tercer trimestre & $\mathrm{S} / 32451$ & $\mathrm{~S} / 7540$ \\
Cuarto trimestre & $\mathrm{S} / 39517$ & $\mathrm{~S} / 32400$ \\
Total & $\mathrm{S} / \mathbf{1 3 5} \mathbf{0 0 6}$ & $\mathrm{S} / \mathbf{5 2} \mathbf{5 6 4}$ \\
\hline
\end{tabular}

Nota: Elaboración propia con base en los registros de cobranza de la empresa Digital San Martín S.A.C. de Tarapoto

Al aplicar una lista de cotejo, se identificó una serie de nudos críticos originados por la ausencia de un sistema de riesgos que informe sobre el récord de pago de los clientes, falta de medidas preventivas del riesgo crediticio, información desactualizada en los registros, deficiente control de los pagos realizados, etc. Esto deja en evidencia el poco interés de la gerencia y ausencia de capacitaciones, programas o talleres que enriquezcan los conocimientos técnicos a favor del personal sobre las labores que desempeñan. Independientemente del contexto presentado por el COVID-19, se percibe un aumento considerable del importe de las cuentas incobrables desde la creación de la organización, registrándose en el primer trimestre un cobro por S/ 4210 de S/ 28521 (Ver Tabla 2); por lo que es primordial la implementación de un sistema de riesgo para estudiar detalladamente la conducta crediticia de los clientes.

Este punto se relaciona con la investigación realizada por Vicente (1995), quien alude que solo el $72 \%$ de las organizaciones colombianas envían mensajes o realizan llamadas como mecanismos de cobro, mientras que el $13 \%$ emite notificaciones a fin de que los clientes morosos sean registrados en el sistema de riesgos, y un $22 \%$ no determina una sanción o emite una papeleta judicial. De la misma manera, el estudio desarrollado por García y Pizzan (2020) se asemeja a los resultados alcanzados, dado que llegaron a concluir que una empresa servicio carece de lineamientos y políticas que especifiquen a detalle los recursos a emplear, los procesos a realizar y los tiempos que se deben desarrollar, así también, carecen de revisión y seguimiento continuo lo cual, ha ocasionado el incremento de las cuentas incobrables.

\subsection{Morosidad en la empresa Digital San Martín S.A.C. de Tarapoto}

Para proceder a clasificar a los clientes calificados como morosos considerando el plazo de demora en el pago, fue indispensable solicitar el registro ordenado y actualizado de cobranza de la organización (Ver Tabla 3). 
Tabla 3

Clasificación de morosos

\begin{tabular}{lccccc}
\hline $\begin{array}{c}\text { Tipo de } \\
\text { morosos }\end{array}$ & $\begin{array}{c}\text { Días de } \\
\text { retraso }\end{array}$ & $\begin{array}{c}\text { Periodo 2019 } \\
\text { Cantidad de } \\
\text { clientes }\end{array}$ & $\begin{array}{c}\text { Monto de la } \\
\text { deuda }\end{array}$ & $\begin{array}{c}\text { Periodo 2020 } \\
\text { Cantidad de } \\
\text { clientes }\end{array}$ & $\begin{array}{c}\text { Monto de la } \\
\text { deuda }\end{array}$ \\
\hline Normal & 8 & 32 & S/ 11200 & 24 & S/ 9521 \\
Potencial & 9 a 30 & 38 & S/ 32 300 & 41 & S/ 40 521 \\
Deficiente & 31 a 60 & 23 & S/ 19550 & 25 & S/ 24 481 \\
Dudoso & 61 a 120 & 14 & S/ 11900 & 28 & S/ 36 428 \\
Pérdida & 120 a más & 18 & S/ 44721 & 46 & S/ 52 564 \\
Total & & 125 & S/ 119671 & 164 & S/ 163515 \\
\hline
\end{tabular}

Nota: Elaboración propia con base en los registros de cobranza de la empresa Digital San Martín S.A.C. de Tarapoto

Los clientes fueron clasificados en función al tiempo de retraso. De acuerdo con la data expuesta, para el periodo 2019 la organización tenía a su disposición a 125 clientes permanentes, donde la mayor parte de estos tenían un plazo de retraso entre 9 y 30 días, posteriormente de 8 días. Así pues, respecto al importe de la deuda, la que incide en mayor medida en los clientes morosos es aquella considerada como pérdida por un importe de S/ 44721 , seguidamente está el potencial con un importe de S/ 32 300, entre otros que dieron un total de S/ 119671 . Sin embargo, para el periodo 2020, el número de clientes deudores aumentó a 164, incrementando en mayor medida aquellos categorizados como "pérdida" al tener 46, donde el importe de la deuda asciende a S/ 52 564, considerando tal importe como incobrable y provisionándolo como gasto. De la misma forma, se pudo evidenciar que existen 41 clientes que fueron categorizados como "potencial" al presentar un tiempo de retraso entre 9 a 30 días calendario, donde la deuda ascendió a S/ 40521.

Por otro lado, se percibe que para el periodo 2020 existió una morosidad superior, puesto que se presentaron índices de $86 \%$ y $92 \%$ de forma respectiva para el tercer y cuarto trimestre. Mientras que, en el primer y cuarto trimestre de 2019 se obtuvo un índice de $57 \%$ y $69 \%$ de modo proporcional. Por ende, el tercer trimestre fue el que presentó mayores variaciones con un 31\%, mientras que el primer trimestre fue el que menos variación tuvo con un 19\%. En efecto, es oportuno manifestar que estos valores porcentuales son producto de la ausencia de herramientas y mecanismos estratégicos que faciliten el cobro oportuno de las deudas por los servicios brindados al crédito, cuya fecha de vencimiento ya culminó y, por ende, se requieren de su cobro inmediato de tal manera que no pueda llegar a convertirse en una cuenta incobrable, generando perdidas para la organización (Ver Tabla 4).

Tabla 4

Índice de morosidad

\begin{tabular}{lccc}
\hline \multicolumn{1}{c}{ Meses } & 2020 & 2019 & Variaciones \\
\hline Enero - Marzo & $75 \%$ & $57 \%$ & $18 \%$ \\
Abril - Junio & $81 \%$ & $60 \%$ & $22 \%$ \\
Julio - Setiembre & $86 \%$ & $54 \%$ & $31 \%$ \\
Octubre - Diciembre & $92 \%$ & $69 \%$ & $22 \%$ \\
\hline
\end{tabular}

Nota: Variación trimestral de la morosidad

Al llevar a cabo un análisis de la afectación financiera y económica de 2020, se conoce que las ventas de la organización fueron por S/ 459 115, donde S/ 52564 no fueron recuperadas, generándose un importe de S/ 47351 como utilidad. Esto suscitó retrasos mayores a 15 días del pago de gastos a terceros y al personal, lo cual ha conllevado a un desabastecimiento de productos y la reducción en las ventas. Respecto a los servicios prestados al crédito, esto se realiza en aquellos casos en los cuales se comercializan productos como banners, tarjetas, hojas membretadas, etc., en los cuales el cliente tiene 15 días para pagar la deuda; no obstante, como el personal tiene funciones de publicidad y marketing, se necesitan colaboradores para contrastar el pago efectivo, suscitando 
un aumento de las cuentas incobrables que para el 2020 ascendió a S/ 135 006. Sobre las ventas al contado, estas son efectuadas cuando se solicita el servicio de monitoreo de cuenta, diseño de fan page, publicaciones en redes sociales, etc., donde el cliente debe efectuar el pago al momento de firmar el contrato elaborado entre las partes a fin de garantizar que se cumpla los compromisos con terceros en el periodo de tiempo proyectado

Esto es análogo a la afirmación de Royo (2013), quien asevera que la mayoría de las organizaciones pertenecientes al sector financiero alcanza un índice de morosidad equivalente a 0,85 porque no manejan adecuadamente sus operaciones de cobro, por lo que proceden a vender sus deudas a las agencias de cobro para recuperar en la mayor medida de lo posible sus recursos. En cambio, Miranda (2018) dedujo que la empresa en su estudio presentó un nivel de morosidad medio equivalente a $49 \%$ y esto afectó significativamente la continuidad de sus actividades al no poder cubrir los gastos incurridos con terceras personas, pues se percibe que existen numerosos reclamos dirigidos a la organización debido a que no se están realizando los pagos a los proveedores y colaboradores en los plazos de tiempo acordado, impidiendo de este modo su buen desempeño operativo.

El contexto expuesto, conllevó a la falta de cumplimiento en el pago a terceros tales como personal y proveedores y, para asegurar el incremento de los niveles de liquidez, la organización tuvo que recurrir a la solicitud de préstamos con el fin de adquirir los medios necesarios. No obstante, a través de la entrevista desarrollada se reconoció que la organización no analiza ni evalúa minuciosamente las causas que generan un importe elevado de pérdidas registradas porque no tiene a su disposición el talento humano idóneo para cumplir con tales atribuciones de forma pertinente, limitando de esta manera el adecuado desarrollo de cada una de las acciones de cobranza.

\subsection{Determinar la incidencia de los procesos de cobranza en la morosidad de la empresa}

\section{Digital San Martín S.A.C. de Tarapoto}

Al realiza un análisis detallado de los reportes e informes financieros, los datos reflejaron un impacto negativo en vista de que las acciones llevadas a cabo en el marco del cobro repercutieron en el aumento de la morosidad de manera inadecuada e inoportuna, pues se han originado pérdidas ascendentes a S/ 52 564. Esto es semejante a la afirmación de Guevara y Upiachihua (2018), quienes indicaron que las acciones que componen la gestión de cobro de deudas inciden en la empresa de modo negativo por cuanto incrementa los índices de morosidad. Igualmente, Miranda (2018) establece que las medidas estratégicas de cobranza y morosidad se relacionan significativamente porque Sig. $<0,05$, ello refleja que la organización no tiene a su disposición, como parte de su acervo documentario organizacional, los lineamientos o pautas de cobro, mientras que las medidas estratégicas de cobranza no son aplicadas oportunamente dentro de cada uno de los casos estudiados, permitiendo deducir de forma general que las acciones estratégicas para el cobro de las deudas pendientes inciden negativamente en los índices de morosidad de la empresa al impedir la recuperación oportuna de los recursos.

De igual modo, los resultados de Campodónico (2019) concuerdan con los alcanzados en el presente estudio, por cuanto concluye que las actividades de gestión de cobranza se relacionan negativamente con la liquidez. Mientras que Castillo (2018) pone en manifiesto que la gestión de los cobros guarda una estrecha relación con la morosidad puesto que los valores estadísticos son $\mathrm{X}^{2}=0,721$ y $\mathrm{p}<0,001$, que demuestra la existencia de una relación positiva significativa de $51,98 \%$, por lo que se procede a aceptar la hipótesis alterna investigativa. Por último, los procedimientos de cobranza se desarrollan de forma deficiente e inciden negativamente en la morosidad del objeto de estudio pues las operaciones orientadas a recuperar las cuentas incobrables no fueron efectuadas eficiente ni oportunamente, lo cual ocasionó un aumento del índice de morosidad a 92\% para octubre-diciembre. 
Entre los problemas que más prevalecen en la empresa, destaca que se tiene a disposición registros e información documentaria no actualizada, ausencia de un sistema que castigue a los clientes morosos, falta de estrategias de cobro acordes con la realidad de la empresa que impiden su oportuna y continua aplicación, ausencia de un monitoreo organizado y sistemático del importe total de la deuda pendiente de pago, deficiente desarrollo de las actividades de control de las deudas para avalar su pago efectivo, etc.; todo ello permitió evidenciar un $84 \%$ de morosidad y $39 \%$ de cobertura, ambos índices suscitados por el aumento del importe de las cuentas pendientes de pago representado por S/135 006, permitiendo de esta forma que se llegue a concluir que los procesos de cobranza se ejecutan de modo deficiente, y ello ocasiona que tengan una incidencia negativa en la morosidad; en la medida en la que el desarrollo deficiente y poco organizado de los mismos conlleva a que el importe de las deudas incobrables incremente. Si bien se ha demostrado que los diversos procesos de cobranza mal direccionados se han visto reflejados en los niveles de morosidad, es importante recalcar también que la situación de crisis sanitaria ha jugado un papel muy importante dado que ello ha limitado la facilidad para acceder a los recursos económicos y financieros requeridos, tal como manifiesta Periche-Delgado, Ramos-Farroñan y Chamolí-Falcón (2020), quienes determinaron que la morosidad incrementó $0,04 \%$ en el primer trimestre de 2020 y su estimación fue hasta 5\%. En ese sentido, la capacidad de pago se vio reducida drásticamente en casi la totalidad de sectores económicos (Ordóñez, Hernández y Mendieta, 2021).

\section{CONCLUSIÓN}

En cuanto al objetivo general, los procesos de cobranza son deficientes e inciden de manera negativa en la morosidad de la empresa Digital San Martin S.A.C. de Tarapoto, dado que las acciones y actividades que se orientan a la recuperación de las cuentas por cobrar no se cumplen de manera oportuna y con la eficiencia del caso, que ha permitido el crecimiento de los índices de morosidad a un índice de $92 \%$ en el último trimestre. Las deficiencias en los procesos de cobranza en la empresa Digital San Martin S.A.C. de Tarapoto, son: reportes con información desactualizada, falta de aplicabilidad de sistemas de castigo de deuda, estrategias de cobranzas aplicadas de manera inoportuna e infrecuente, no cuenta con un control ordenado del total de las deudas, y no se desarrollan acciones de seguimiento de la deuda. Al evaluar la morosidad en la empresa Digital San Martin S.A.C, se conoció que presenta un índice de morosidad de $84 \%$.

Dentro de las limitaciones presentadas en el estudio, la muestra no fue suficientemente representativa para lograr recolectar mayor información. Así también, el método de la investigación empleado (exploratorio) ha limitado la aplicación estadística en el estudio, por lo que, no se ha realizado el cálculo para la comprobación de la hipótesis.

A raíz de los resultados, como agenda futura de investigación se presentan las siguientes interrogantes: ¿Existen diferencias significativas entre los niveles de morosidad de los sectores económicos en las pymes de San Martín?, ¿Cuáles son las estrategias de mayor eficiencia en para la recuperación de cartera morosa en las empresas sanmartinenses?, y ¿Existirá diferencia significativa entre los niveles de morosidad registradas antes y durante la COVID-19 en las Mypes del Perú?

\section{REFERENCIAS}

Arbaiza, L. (2019). Como elaborar una tesis de grado. ( $\left.1^{\circ} \mathrm{ed}\right)$. Colombia: Esan Ediciones

Bengolea, A., \& Pérez, A. (2016). La ilegalidad del cargo de gestión de cobranza en loscontratos de consumo. Derecho comercial y de las obligaciones: Revista de doctrina, jurisprudencia, legislación y práctica, 5(279), 17-28. https://dialnet.unirioja.es/servlet/articulo?codigo $=5674116$

Campodónico, S. (2019). Gestión de cobranza y liquidez de la empresa Berean ServiceSAC-Tarapoto 2017-2018 (Tesis para obtener el grado de Contador Público). http://repositorio.unsm.edu.pe/handle/11458/3645

Castillo, M. (2018). Gestión de cobranzas y nivel de morosidad en la empresa Limpia Max-Lima 2017 (Tesis para 
obtener el grado de Licenciado en Administración). http://repositorio.uigv.edu.pe/handle/20.500.11818/3262

Do Prado, J., Melo, F., Carvalho, G., \& Ribeiro, A. (2019). Analysis of credit risk faced by public companies in Brazil: an approach based on discriminant analysis, logistic regression and artificial neural networks. Estudios Gerenciales, 35(153),56-89. https://doi.org/10.18046/j.estger.2019.153.3151

Ferro, J. M. (2014). Morosidad, deudores y Outsourcing. D.F: CreateSpace Independent Publishing Platform

García, A. (2018). Teoría y metodología de la investigación científica. ( $\left.1^{\circ} \mathrm{ed}\right)$. Colombia: Alfaomega Colombiana

García, E. \& Pizzan, N. (2020). Procesos de cobranza y su incidencia en la morosidad de la empresa Digital San Martin S.A.C. de Tarapoto, año 2020. Universidad Cesar Vallejo. Tarapoto. https://n9.cl/zlu51v

García, X., Galarza, C., \& Grijalva, G. (2019) La gestión de créditos y cobranzas caso empresa "Multillanta Ramírez". Revista de Investigación, Formación y Desarrollo, 7(1), 73-81. https://dialnet.unirioja.es/servlet/articulo?codigo $=7299581$

Gonzáles, A., Leal, L., Martínez, D., \& Morales, D. (2019). Herramientas para la gestiónpor procesos. Cuadernos Latinoamericanos de Administración, 15(28), 42-89. https://www.redalyc.org/articulo.oa?id=409659500003

Gonzalo, J. (2012). Los efectos económicos y financieros de la morosidad en la empresa. Estrategia financiera, (290), $8-13$.

https://dialnet.unirioja.es/servlet/articulo?codigo $=3809823$

Guevara, M, \& Upiachihua, M. (2018). Gestión cobranza y morosidad de la empresa Televisión SAC. Tarapoto, año 2017 (Tesis para obtener el grado de Contador Público). https://repositorio.ucv.edu.pe/handle/20.500.12692/35263

Guillén, E., \& Peñafiel, L. (2018). Modelos predictores de la morosidad con variables macroeconómicas. Revista Ciencia UNEMI, 11(26), 13-24. https://doi.org/10.29076/issn.2528-7737vol11iss26.2018pp13-24p

Guizar, I., González, D., \& Ezzahra, F. (2020). Participación en el mercado de créditoformal versus el informal en México. Revista Científica Multidisciplinaria de Prospectiva, 27(2), 56-89. https://doi.org/10.30878/ces.v27n2a2

Landeta, J., \& Ynzunza, C. (2017). El impacto del crédito y la cobranza en las utilidades.Revista Poliantea, 13(24), 47-62. https://dialnet.unirioja.es/servlet/articulo?codigo $=6160650$

Lionel, A. (2018). Gestión estratégica de cobranzas. Santiago: Ediciones UC.

López, A. (2006). El impacto de la morosidad. Molinería y panadería. Revistaprofesional de panadería y pastelería, 52(1155), 60-61. https://dialnet.unirioja.es/servlet/articulo?codigo $=2007226$

López, A. (2019). Pasos de la gestión de cobranza telefónica. Contact Center CallCenter \& IP solutions, (97), 5254. https://contactcentersonline.com/revistas/revista-n97/

Magallanes, J. (2019) Crisis económica y estabilidad bancaria en el Perú 2007-2015._Anales Científicos, 77(2), 298-308. http://dx.doi.org/10.21704/ac.v77i2.703

Mauad, P. (2017). Aplicaciones para la gestión de crédito y cobro de clientes._Estrategia financiera, 42 (237), 48-51. https://dialnet.unirioja.es/servlet/articulo? codigo $=2241553$

Miranda, R. (2018). Estrategia de cobranza y morosidad de los clientes de la empresaTalma, Callao-2017 (Tesis para obtener el grado de Licenciada en Administración). https://repositorio.ucv.edu.pe/handle/20.500.12692/23257

Ordóñez, J., Hernández, A., \& Mendieta, P. (2021). Índice de morosidad en la banca ecuatoriana durante la pandemia del COVID-19. Centro Sur. Social Science Journal, (2), 19-29.

https://www.centrosureditorial.com/index.php/revista/article/view/193

Periche-Delgado, G.S., Ramos-Farroñan, E. V., \& Chamolí-Falcón, A. W. (2020). La morosidad ante un confinamiento del Covid-19 en la Caja Rural de Ahorro y Crédito Raíz, Perú. Investigación Valdizana, 14(4), 206-212. https://doi.org/10.33554/riv.14.4.801

Núñez, J. (1999). La morosidad, causas y efectos. Estrategia financiera, 8(153), 16-20. https://dialnet.unirioja.es/servlet/articulo?codigo $=156255$

Ramos, J. (2020) La morosidad, causas y efectos. Estrategia financiera. Vol.8(153), pp. 16-20. https://dialnet.unirioja.es/servlet/articulo?codigo $=156255$

Rodrigues, R, \& Florencio, J. (2020). Income smoothing practices and conservatism inbrazilian credit unions. Revista Pensamento Contemporâneo em Administração,14(1), 76-88. https://doi.org/10.12712/rpca.v14i1.38886

Romero, A. (2017). La lucha contra la morosidad y el fraude fiscal en España continúa:el nuevo procedimiento de la agencia tributaria para embargar depósitos bancarios por internet. Revista Aranzadi Doctrinal, 11(2), 13-26. https://dialnet.unirioja.es/servlet/articulo?codigo $=5893070$

Royo, B. (2013). La gestión de la morosidad en entidades financieras. Actuarios, 15(33), 54-56. https://dialnet.unirioja.es/servlet/articulo?codigo $=4531687$

Vicente, J. (1995) Estrategias para combatir la morosidad. Estrategia financiera, 112, 32-36. https://dialnet.unirioja.es/servlet/articulo? $\operatorname{codig} 0=155909$ 


\section{Anexo 1. Lista de cotejo}

El presente instrumento tiene como finalidad captar hechos o hallazgos relevantes que demuestran las deficiencias en los procesos de cobranzas de la empresa Digital San Martin SAC.

\begin{tabular}{|c|c|c|c|}
\hline \multirow{2}{*}{$\begin{array}{c}\text { Dimensión: Identificación del riesgo } \\
\text { Indicador: Conocimiento del cliente }\end{array}$} & \multicolumn{2}{|c|}{$\begin{array}{l}\text { Cumplimiento } \\
\text { de las acciones }\end{array}$} & \multirow[t]{2}{*}{$\begin{array}{c}\text { Observación / } \\
\text { Evidencia }\end{array}$} \\
\hline & $\mathrm{Si}$ & No & \\
\hline \multicolumn{4}{|l|}{$\begin{array}{l}\text { 1. Se solicitan reportes (Historial crediticios), para conocer } \\
\text { la situación económica del cliente }\end{array}$} \\
\hline \multicolumn{4}{|l|}{$\begin{array}{l}\text { 2. ¿Se aplican sistemas de riesgos para conocer el record de } \\
\text { pagos }\end{array}$} \\
\hline \multicolumn{4}{|l|}{ 3. Se desarrollan acciones para prevenir el riesgo crediticio } \\
\hline \multicolumn{4}{|l|}{ Indicador: Identificación de la capacidad de pago } \\
\hline \multicolumn{4}{|l|}{$\begin{array}{l}\text { 4. Se especifica en los reportes la capacidad de pago del } \\
\text { cliente }\end{array}$} \\
\hline \multicolumn{4}{|l|}{ 5. Se especifica información actual del cliente } \\
\hline \multicolumn{4}{|l|}{ Indicador: Seguimiento de pagos. } \\
\hline \multicolumn{4}{|l|}{$\begin{array}{l}\text { 6. Se efectúa el seguimiento continuo de los pagos } \\
\text { detallados en el cronograma de pagos }\end{array}$} \\
\hline \multicolumn{4}{|l|}{ Dimensión: Aplicación de las estrategias de cobranzas } \\
\hline \multicolumn{4}{|l|}{ Indicador: Mensajes } \\
\hline \multicolumn{4}{|l|}{$\begin{array}{l}\text { 7. Se efectúan mensajes como aviso del vencimiento de la } \\
\text { deuda }\end{array}$} \\
\hline \multicolumn{4}{|l|}{$\begin{array}{l}\text { 8. Existe un responsable de efectuar los mensajes de manera } \\
\text { continua }\end{array}$} \\
\hline \multicolumn{4}{|l|}{ Indicador: Llamadas } \\
\hline \multicolumn{4}{|l|}{$\begin{array}{l}\text { 9. Se efectúan llamadas como estrategia para recuperar las } \\
\text { deudas }\end{array}$} \\
\hline \multicolumn{4}{|l|}{ Indicador: Notificaciones } \\
\hline \multicolumn{4}{|l|}{ 10. Se elaboran notificaciones para exigir la deuda } \\
\hline \multicolumn{4}{|l|}{ Indicador: Sistemas de cobranza } \\
\hline 11. Se emplean sistemas de cobranzas & & & \\
\hline
\end{tabular}




\section{Anexo 2. Entrevista}

La investigación, tiene como objetivo evaluar los procesos de cobranza en una empresa de servicios de marketing, para ello se solicita responder con objetividad y veracidad, a fin de obtener resultados trasparentes y verdaderos

\section{DATOS}

Nombre del entrevistado:

Cargo:

Tiempo de labor:

Departamento:

Fecha de la entrevista:

\section{Dimensión: Identificación del riesgo \\ Indicador: Conocimiento del cliente}

P1. ¿Cuáles son las actividades efectuadas en la identificación del riesgo?

P2. ¿Qué sistema aplican para conocer los records de pagos, y quienes se encargan de tal actividad?

P3. ¿Cuáles son los registros o reportes que solicitan para conocer al cliente?

Indicador: Identificación de la capacidad de pago

P4. ¿Que se detallan en los reportes que evalúan al cliente?

P5. ¿Por qué es importante conocer la información personal del cliente?

Indicador: Seguimiento de pagos.

P6. ¿Cada cuánto tiempo se realiza el seguimiento de los pagos?

P7. ¿Qué acciones efectúan para limitar conductas que pueden generar la incobrabilidad de las cuentas por cobrar?

\section{Dimensión: Aplicación de las estrategias de cobranzas}

\section{Indicador: Mensajes}

P8. ¿A qué tipo de deudor se aplica esta estrategia?

P9. ¿Cómo se desarrolla la aplicación de las estrategias?

\section{Indicador: Llamadas}

P10. ¿A qué tipo de deudor se aplica esta estrategia?

P11. ¿¿Cuántas llamadas se realiza al cliente?

\section{Indicador: Notificaciones}

P12. ¿A qué tipo de deudor se aplica esta estrategia?

P13. ¿Cuál es la cantidad máxima en la emisión de las notificaciones?

\section{Indicador: Sistemas de cobranza}

P14. ¿Cuántos morosos cuenta hasta la fecha?

P15. ¿En qué consiste el sistema de cobranzas? 
Anexo 3. Guía de análisis documental.

El presente instrumento tiene como finalidad recolectar datos informáticos que detallen la cantidad de morosos y el índice de morosidad

\begin{tabular}{|c|c|c|c|c|c|}
\hline \multirow{4}{*}{$\begin{array}{c}\text { Documentos } \\
\text { a emplear }\end{array}$} & \multicolumn{4}{|c|}{ Morosidad - PERIODO 2020 } \\
\cline { 2 - 6 } & Clasificación & Cantidad & Montos & \multicolumn{1}{|c|}{$\begin{array}{c}\text { Índice de } \\
\text { morosidad }\end{array}$} & $\begin{array}{c}\text { índice de } \\
\text { cobertura }\end{array}$ \\
\hline \multirow{4}{*}{$\begin{array}{c}\text { Reportes de } \\
\text { cobranzas }\end{array}$} & Normal & & & & \\
\cline { 2 - 6 } & Peficientes & & & & \\
\cline { 2 - 6 } & Dudoso & & & & \\
\cline { 2 - 6 } & Perdida & & & & \\
\cline { 2 - 6 } & & & & & \\
\hline
\end{tabular}

